\title{
Vibration Response Imaging in Idiopathic Pulmonary Fibrosis: A Pilot Study
}

\author{
Qing-xia Liu MMed, Wei-jie Guan PhD, Yan-qing Xie PhD, Jia-ying An MB, \\ Mei Jiang PhD, Zheng Zhu MMed, E Guo MMed, Xin-xin Yu MB, \\ Wen-ting Liu MB, Yi Gao MMed, and Jin-ping Zheng MD
}

\begin{abstract}
BACKGROUND: Vibration response imaging (VRI) is a novel imaging technique and little is known about its characteristics and diagnostic value in idiopathic pulmonary fibrosis (IPF). The aim of this study was to investigate the features of VRI in subjects with IPF. METHODS: We enrolled 23 subjects with IPF (42-74 y old) and 28 healthy subjects (42-72 y old). Subjects with IPF were diagnosed by lung biopsy and underwent VRI, spirometry, lung diffusion testing, and chest $\mathrm{x}$-ray or computed tomography, which entailed assessment of the value of VRI indices. RESULTS: The total VRI score correlated statistically with single-breath carbon monoxide diffusing capacity percent predicted $(r=-0.30, P=.04)$, but not with $F V C$ percent predicted, $F E V_{1}$ percent predicted, and $\mathrm{FEV}_{1} / \mathrm{FVC}(\mathrm{r}=-0.27,-0.22$, and 0.19 ; all $P>.05)$. Compared with healthy subjects $(17.9 \%), 20$ subjects with IPF $(86.96 \%, P<.01)$ presented with significantly increased crackles. The difference in quality lung data in all lung regions was unremarkable (all $P>.05$ ), except for the upper right and lower left lobes $(P<.05)$. Overall, VRI parameters yielded acceptable assay sensitivity and specificity. Maximum energy frame was characterized by the highest diagnostic value (sensitivity, 1.00; specificity, 0.82 ), followed by presence of abundant crackles (sensitivity, 0.70; specificity, 0.96). Total VRI score was not a sensitive indicator of IPF, owing to low assay sensitivity (0.70) and specificity (0.64). CONCLUSIONS: VRI may be helpful to discriminate between IPF subjects and healthy individuals. Maximum energy frame and abundant crackles might serve as a diagnostic tool for IPF. Key words: idiopathic pulmonary fibrosis; pulmonary function; pulmonary breathing imaging diagnosis system; vibration response imaging. [Respir Care 2014;59(7):1071-1077. (C) 2014 Daedalus Enterprises]
\end{abstract}

\section{Introduction}

Idiopathic pulmonary fibrosis (IPF), characterized by dyspnea on exertion, hypoxia, restrictive ventilatory dys-

\footnotetext{
Dr Q Liu is affiliated with the State Key Laboratory of Respiratory Disease, First Affiliated Hospital of Guangzhou Medical University, Guangzhou, Guangdong, People's Republic of China and Qingyuan People's Hospital, Qingyuan, Guangdong, People's Republic of China. Drs Guan, Xie, An, Jiang, Zhu, Guo, Yu, W Liu, Gao, and Zheng are affiliated with the State Key Laboratory of Respiratory Disease, First Affiliated Hospital of Guangzhou Medical University, Guangzhou, Guangdong, People's Republic of China.
}

This study was supported by National Key Technology Research and Development Program (2012BAI05B01/2013BAI09B09).

Supplementary material related to this paper is available at http:// www.rcjournal.com. function, reduced diffusing capacity, and pulmonary fibrosis on chest computed tomography, has been increasingly recognized as a diversity of disorders involving the pulmonary interstitium and/or parenchyma. The accepted standard of diagnosis of IPF relies on lung biopsy, and diag-

\footnotetext{
Drs Q Liu and Guan are co-first authors.

The authors have disclosed no conflicts of interest.

Correspondence: Jin-ping Zheng MD, State Key Laboratory of Respiratory Disease, National Clinical Research Center for Respiratory Disease, First Affiliated Hospital of Guangzhou Medical University, 151 Yanjiang Road, Guangzhou, Guangdong 510120, People's Republic of China. Email: jpzhenggy@163.com.
}

DOI: $10.4187 /$ respcare.02739 
nosis can be achieved by chest imaging and lung function tests. ${ }^{1}$ In addition, the fact that measurement of diffusing capacity failed to identify the presence of $\mathrm{IPF}^{2}$ suggested that conventional invasive measures might have limited significance. In this regard, development of a novel noninvasive technique with improved diagnostic power is urgently indicated.

Vibration response imaging (VRI) is a novel technique in which the diagnostic information is derived from the vibration energy superimposed on the respiratory cycles. The turbulent air and vibration generated within the airways can be sensitively captured by the sensors, thus allowing for a noninvasive, radiation-free, and convenient approach to be clinically applied. The VRI measurement has been increasingly applied for the diagnosis of respiratory diseases, including COPD, ${ }^{3,4}$ asthma, ${ }^{5}$ airway foreign body, ${ }^{6}$ pleural effusion, ${ }^{7}$ and pneumonia. ${ }^{8}$ Furthermore, VRI plays a role in the assessment of lung function in regions of interest, ${ }^{9}$ intervention treatment of pulmonary diseases, ${ }^{10}$ screening of candidates for lung surgery, ${ }^{11}$ and monitoring of postintubation conditions. ${ }^{10}$ Unfortunately, whether VRI has a high diagnostic value for IPF remains has not been sufficiently studied. We hypothesized that subjects with IPF may have distinct characteristics compared with healthy subjects and that the major VRI indices, as previous studies suggested, are useful in the diagnosis of IPF.

Consequently, we sought to determine the characteristics and diagnostic value of VRI in subjects with IPF, thereby offering a rationale for clinical application.

\section{Methods}

\section{Subjects}

We recruited 51 subjects, including 23 subjects with IPF (42-74 y old) from the out-patient clinics and 28 healthy subjects (42-72 y old) from the health check-up center of the First Affiliated Hospital of Guangzhou Medical University, between September 2011 and January 2012. The inclusion criteria for subjects with IPF comprised (1) subjects of either sex with IPF, ${ }^{12}$ (2) pathologic characteristics of IPF as clinically diagnosed by typical chest computed tomography, and (3) absence of miscellaneous severe systemic diseases. Those subjects with rib cage or spinal deformity, skin lesions, or limited understanding were excluded.

The inclusion criteria for healthy subjects comprised: (1) nonsmokers of either sex, (2) normal chest radiograph, (3) normal spirometry and diffusing capacity, (4) absence of upper respiratory tract infection within 4 weeks, and (5) no evidence of other chronic cardiopulmonary diseases. All subjects gave written informed consent prior to the study. This study protocol was approved by the ethics committee of the First Affiliated Hospital of Guangzhou Medical University (protocol 2012-26).

\section{QUICK LOOK}

\section{Current knowledge}

Vibration response imaging (VRI) is a novel technique in which the diagnostic information is derived from the vibration energy superimposed on the respiratory cycles. The turbulent air and vibration generated within the airways are captured by sensors, allowing for a noninvasive, radiation-free way to evaluate lung disease. The clinical use of VRI is not routine.

\section{What this paper contributes to our knowledge}

VRI was able to discriminate between subjects with idiopathic pulmonary fibrosis and healthy individuals. The maximum energy frame during VRI along with abundant crackles could be used as a diagnostic tool for idiopathic fibrosis.

\section{Study Protocol}

This was a single-center study. All consecutive clinically stable subjects (characterized by no significant change $[<20 \%]$ in the frequency of cough, sputum volume, spirometry, and degree of dyspnea) underwent chest x-ray/clinical diagnosis by typical chest computed tomography, VRI, spirometry, and measurement of diffusing capacity, in this order. Biopsy was scheduled when clinically stable, at least 3 months after the initial screening. For healthy subjects, the need to perform chest $\mathrm{x}$-ray was waived. These subjects required subsequent analysis on the VRI indices.

\section{Lung Function Testing}

Spirometry (Quark 4, COSMED Co Ltd, Rome, Italy) and measurement of diffusing capacity using the singlebreath carbon monoxide washout method were conducted according to the 2005 guidelines of the American Thoracic Society and the European Respiratory Society. ${ }^{13,14}$

Briefly, FVC, $\mathrm{FEV}_{1}$, FVC percent predicted, $\mathrm{FEV}_{1}$ percent predicted, and $\mathrm{FEV}_{1} / \mathrm{FVC}$ were analyzed by spirometry. At least 3 (no more than 8) maneuvers were performed, with variations between the best 2 maneuvers of $<5 \%$ or $150 \mathrm{~mL}$ in $\mathrm{FVC}$ and $\mathrm{FEV}_{1}$. The maximum FVC and $\mathrm{FEV}_{1}$ values were reported.

The carbon monoxide diffusing capacity $\left(\mathrm{D}_{\mathrm{LCO}}\right)$ and the single-breath $\mathrm{D}_{\mathrm{LCO}}$ percent predicted were analyzed by the single-breath carbon monoxide washout technique. The interval between 2 consecutive measurements was not less than $4 \mathrm{~min}$, and the variation coefficient for single-breath $\mathrm{D}_{\mathrm{LCO}}$ should be $<10 \%$ or $3 \mathrm{~mL} / \mathrm{min} / \mathrm{mm} \mathrm{Hg}$. Single-breath $\mathrm{D}_{\mathrm{LCO}}$ was reported based on the mean of the 2 measurements. 


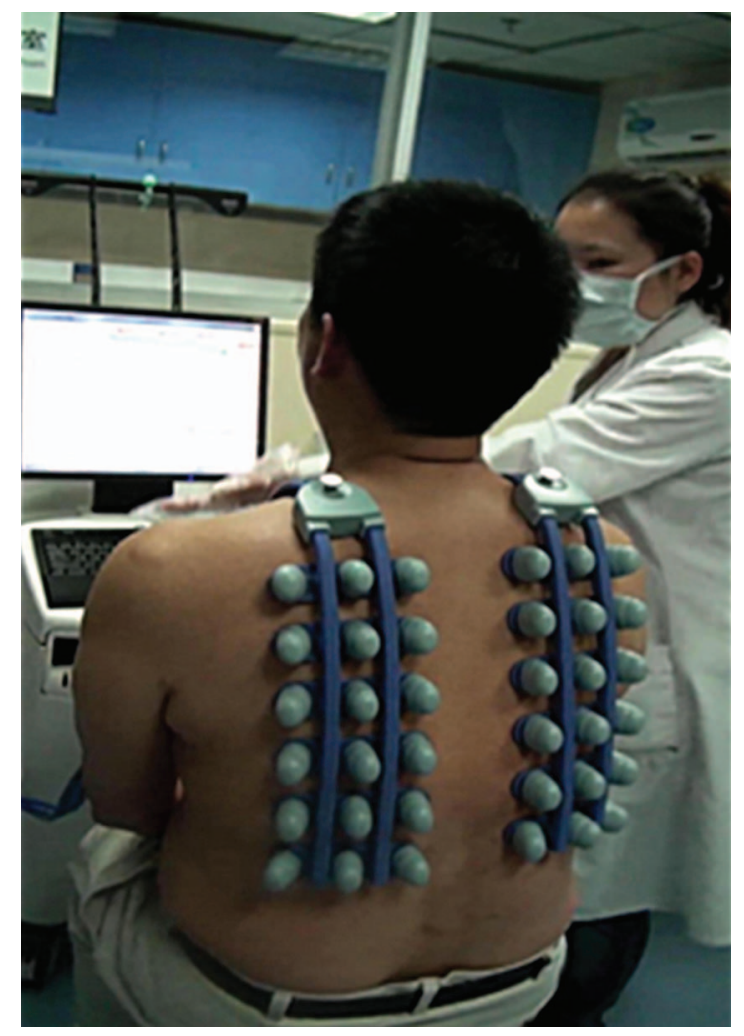

Fig. 1. Placement of vibration response imaging.

\section{Brief Overview of VRI}

By employing an array of specific sensors placed in an orderly manner on the pulmonary projection regions of the subject's back, the VRI lung system (Deep Breeze Ltd, Or-Akiva, Israel) is capable of capturing the changes in vibration, as reflected by the constantly altered air flow (Fig. 1). This entails simulation of primitive mechanical signals that allow transformation into digital signals for deriving the band-pass filtered images based on immediate sampling, thus reflecting dynamic variation in the breathing cycles. The major indices and their significance are introduced in the VRI analysis section (see below and the supplementary materials at http://www.rcjournal.com).

\section{Critiques of VRI}

VRI testing was performed in a quiet, noise-free chamber. After a 5-min resting period, subjects were seated, and 2 sensor arrays were placed on their backs. These sensor arrays were placed bilaterally in parallel at the longitudinal and vertical axes $( \pm 1.0 \mathrm{~cm})$ and at least $2.5 \mathrm{~cm}$ away from the spine. The top-row sensors were positioned $1.5-2.0 \mathrm{~cm}$ superior to the shoulder blade. Subjects were instructed to breathe normally via the mouth during a 12 -s recording (3-5 respiratory cycles). Forced exhalation or miscella- neous artifacts should be avoided. Subsequent tests were conducted at 1-2-min intervals. This was followed by selection of a smooth vibration energy curve and maximum energy frame (MEF) image for further analysis. Test tolerability was assessed by inquiry about subject discomfort during measurement.

\section{VRI Analysis}

VRI analysis was conducted according to the vibration energy graph, dynamic image, MEF, quality lung data (QLD), envelope of acoustic signal (EVP), and presence of wheezes or crackles, for which definitions are provided. The images of each individual were rated, for calculation of the total score, based on the features as outlined. (See online supplementary material.) The normal VRI images are shown Figure 2.

\section{Statistical Analysis}

Data were analyzed using SPSS 16.0 (SPSS, Chicago, Illinois). Continuously distributed variables were presented as mean \pm SD following test of normality. The Student $t$ test or chi-square test was used to compare the differences between both groups, depending on the distribution pattern of variables. Spearman correlation analysis was applied to analyze the correlation between VRI and spirometric parameters and diffusing capacity. The diagnostic performance of VRI total score, MEF, and presence of crackles was compared. $P<.05$ was defined as statistically significant.

\section{Results}

\section{Baseline Demographic Characteristics}

Subjects with IPF did not differ statistically from healthy subjects in terms of age $(P=.82)$ and height $(P=.18)$. The differences in weight and male-to-female ratio were significant (both $P<.05$ ) but not clinically important. Lung function parameters, including FVC $(P<.01)$, FVC percent predicted $(P<.01), \mathrm{FEV}_{1}$ percent predicted $(P<.01)$, and single-breath $\mathrm{D}_{\mathrm{LCO}}$ percent predicted $(P<.01)$, in IPF subjects were significantly lower than those in healthy subjects, with the exception of a higher $\mathrm{FEV}_{1} / \mathrm{FVC}(P<.01)$ (Table 1$)$.

\section{Dynamic Image}

The VRI total score correlated with single-breath $\mathrm{D}_{\mathrm{LCO}}$ percent predicted ( $\mathrm{r}=-0.30, P=.04$ ) (Fig. 3), but not with FVC percent predicted $(\mathrm{r}=-0.27, P=.06), \mathrm{FEV}_{1}$ percent predicted $(\mathrm{r}=-0.22, P=.13)$, and $\mathrm{FEV}_{1} / \mathrm{FVC}$ $(\mathrm{r}=0.19, P=.19)$. 


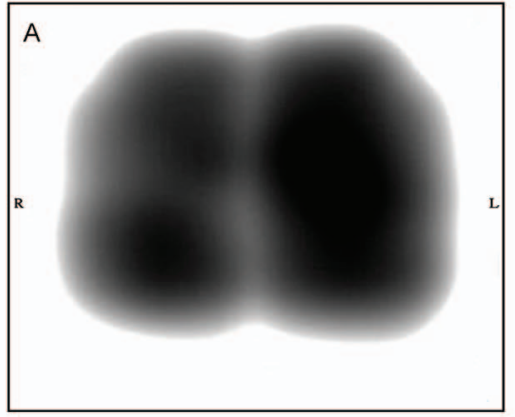

\begin{tabular}{|l|c|c|}
\hline \multirow{2}{*}{$B \quad$ Calculated for frames from 56 to 71} \\
\cline { 2 - 3 } & Right & Left \\
\hline Upper & $12 \%$ & $14 \%$ \\
\hline Middle & $14 \%$ & $17 \%$ \\
\hline Lower & $19 \%$ & $24 \%$ \\
\hline Total & $45 \%$ & $55 \%$ \\
\hline
\end{tabular}

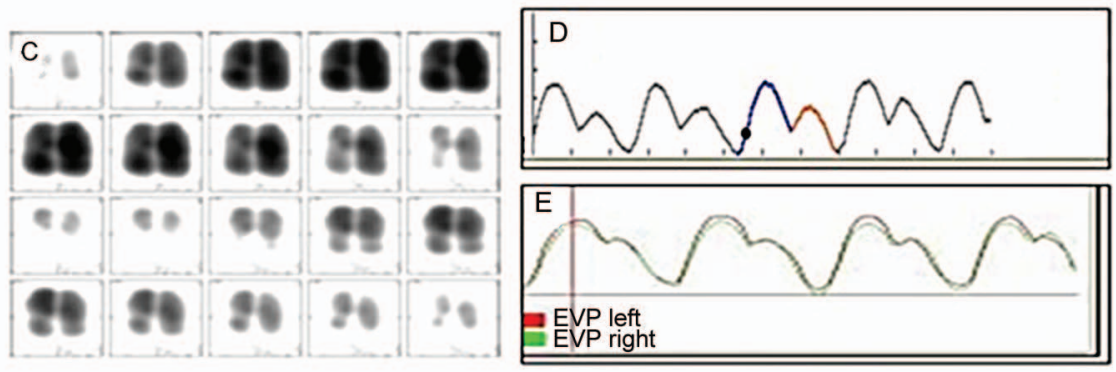

Fig. 2. Schematic diagrams of normal vibration response images. A: Maximum energy frame image showing typical distribution of vibration energy as reflected by the grayscale. B: Quality lung data image calculated for frames $56-71$, showing distribution of vibration energy at different pulmonary fields. C: Dynamic image showing synchronized vibrations without evidence of bouncing. D: Vibration energy graph showing similar individual breathing cycle. E: Envelope of acoustic signal (EVP) image showing synchronized vibrations with equal amplitude.

Table 1. Baseline Characteristics

\begin{tabular}{|c|c|c|c|c|}
\hline Characteristic & $\begin{array}{c}\text { Healthy } \\
\text { Subjects } \\
(n=28), \\
\text { Mean } \pm \text { SD }\end{array}$ & $\begin{array}{c}\text { IPF } \\
\text { Subjects } \\
(n=23), \\
\text { Mean } \pm \text { SD }\end{array}$ & Statistics & $P$ \\
\hline Age (y) & $55.86 \pm 1.71$ & $56.43 \pm 1.86$ & -0.23 & .82 \\
\hline Height $(\mathrm{cm})$ & $157.5 \pm 1.47$ & $160.52 \pm 1.61$ & -0.14 & .18 \\
\hline Weight (kg) & $58.05 \pm 1.89$ & $65.94 \pm 1.63$ & -3.09 & $<.01$ \\
\hline Male/female ratio $(n)$ & $7 / 21$ & $13 / 10$ & $-2.27 *$ & .02 \\
\hline $\mathrm{FVC}(\mathrm{L})$ & $2.82 \pm 0.12$ & $2.16 \pm 1.53$ & 3.52 & $<.01$ \\
\hline FVC $\%$ predicted & $103.68 \pm 2.15$ & $68.45 \pm 3.43$ & 9.02 & $<.01$ \\
\hline $\mathrm{FEV}_{1}(\mathrm{~L})$ & $2.20 \pm 0.11$ & $1.86 \pm 0.13$ & 0.05 & .34 \\
\hline $\mathrm{FEV}_{1} \%$ predicted & $99.69 \pm 1.88$ & $71.81 \pm 3.54$ & -5.32 & $<.01$ \\
\hline $\mathrm{FEV}_{1} / \mathrm{FVC}$ & $0.80 \pm 0.01$ & $0.86 \pm 0.01$ & -3.59 & $<.01$ \\
\hline $\begin{array}{l}\text { Single-breath } \mathrm{D}_{\mathrm{LCO}} \\
\% \text { predicted }\end{array}$ & $88.81 \pm 2.18$ & $44.68 \pm 3.74$ & 10.63 & $<.01$ \\
\hline Total score & $15.57 \pm 0.55$ & $17.17 \pm 0.49$ & -2.15 & .03 \\
\hline $\begin{array}{l}\text { Maximum vibration } \\
\text { energy }\end{array}$ & $1.91 \pm 0.07$ & $1.72 \pm 0.06$ & 1.93 & .06 \\
\hline
\end{tabular}

* For the chi-square test; the Student $t$ test was applied for all other comparisons. $\mathrm{IPF}=$ idiopathic pulmonary fibrosis

$\mathrm{D}_{\mathrm{LCO}}=$ carbon monoxide diffusing capacity

\section{Features of MEF Image}

The MEF image of healthy subjects evidenced a comparatively even distribution of the vibration energy, as reflected by the gradient of grayscales. The grossly sym-

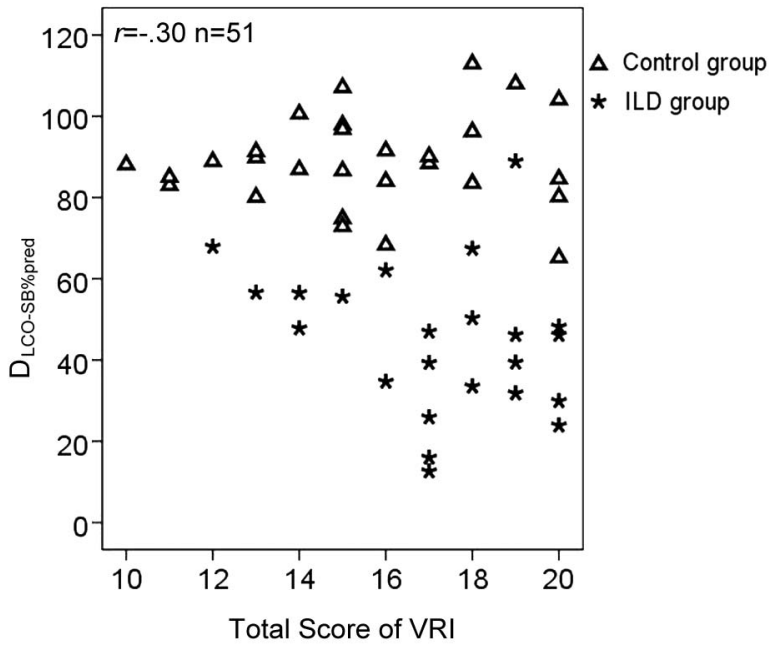

Fig. 3. Correlation between diffusing capacity of carbon monoxide percent predicted measured by the single-breath method ( $D_{\text {LCO-SB\%pred }}$ ) and the vibration response imaging (VRI) total score. $\Delta=$ controls; * $=$ interstitial lung disease (ILD) group.

metric pattern of the vibration energy could be noted bilaterally (Fig. 4A). In contrast, subjects with IPF demonstrated a totally different pattern from healthy subjects. This included bilaterally constrained regions of vibration energy, triangle-like distribution of maximum energy that was restricted to and intensified in the lower lobes, and weakened intensity of vibration energy in the upper lobes (Fig. 4B). Such features were not found in 


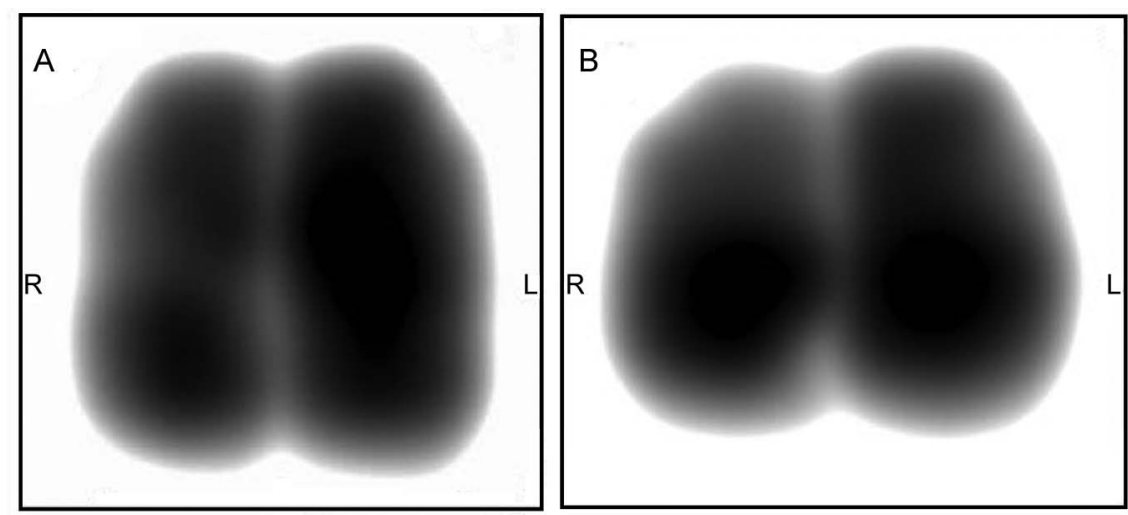

Fig. 4. Maximum energy frame (MEF) images. A: MEF of a healthy female subject. B: MEF of a female subject with interstitial lung disease.

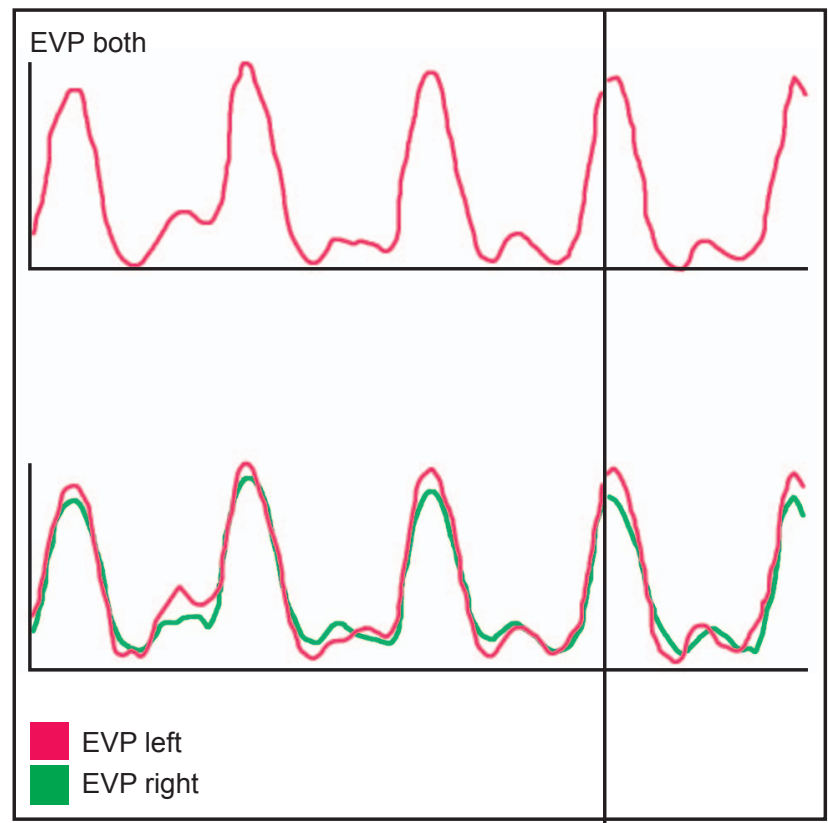

Fig. 5. Envelope of acoustic signal (EVP) curve in a female subject with interstitial lung disease.

miscellaneous respiratory diseases, ie asthma and COPD (data not shown).

\section{EVP Image}

Like healthy subjects, subjects with IPF demonstrated a slight inconsistency between EVP signals in the right and left lungs (Fig. 5).

\section{Adventitious Lung Sounds}

Twenty subjects with IPF $(20 / 23,87 \%)$ presented with significantly increased crackles (blue dots in Fig. 6A) compared with healthy subjects, of whom $5(17.9 \%, P<.01)$ presented with minor crackles (Fig. 6B).

\section{Quality Lung Data}

The difference in QLD in all lung regions was unremarkable (all $P>.05$ ), except for the upper right and lower left lobes $(P=.01)$ (Table 2).

\section{Diagnostic Power of Major VRI Indices}

Overall, VRI parameters yielded acceptable assay sensitivity and specificity. MEF was characterized by the highest diagnostic value, followed by presence of abundant crackles. The VRI total score did not appear to be a sensitive marker indicative of IPF. The diagnostic indices of EVP and QLD could not be derived and were therefore not analyzed in this study (Table 3).

\section{Discussion}

Our data showed that, compared with vibration energy images in healthy subjects (see Fig. 2), IPF subjects were characterized by bilaterally constrained vibration energy, triangle-like distribution of maximum energy that was restricted to and intensified in the lower lobes, and weakened intensity of vibration energy in the upper lobes. There was a positive correlation between the VRI total score and single-breath $\mathrm{D}_{\mathrm{LCO}}$ percent predicted. IPF subjects also had an increased number of crackles as the adventitious lung sounds compared with healthy subjects. MEF and presence of abundant crackles were the VRI indices with highest diagnostic value.

IPF comprises a diversity of diseases characterized by various clinical and imaging features. Typically, restrictive ventilatory dysfunction (reduced $\mathrm{FVC}$ and $\mathrm{FEV}_{1}$ yet normal $\mathrm{FEV}_{1} / \mathrm{FVC}$ ) and reduced diffusing capacity (low single-breath $\mathrm{D}_{\mathrm{LCO}}$ ) may be readily identified in most subjects, particularly those with increased disease severity. Although there is limited literature regarding the detailed interpretation of changes observed in VRI indices, it is 


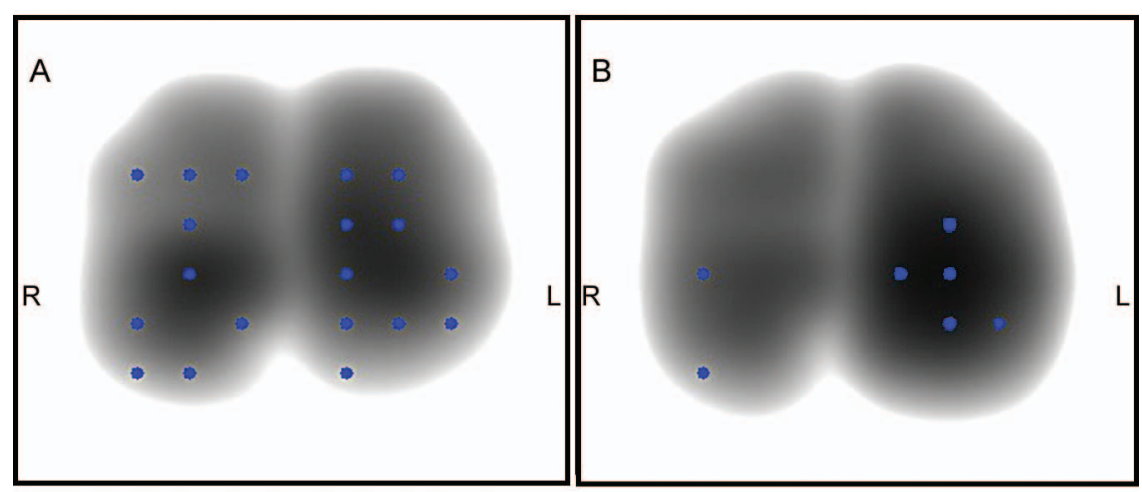

Fig. 6. Adventitious lung sound. A: abundant crackles (blue dots) in a female subject with interstitial lung disease. B: minor crackles (blue dots) in a healthy female subject.

Table 2. Comparison of QLD in Both Groups

\begin{tabular}{lccc}
\hline \hline \multirow{2}{*}{$\begin{array}{c}\text { Lung } \\
\text { Region }\end{array}$} & $\begin{array}{c}\text { Healthy Group } \\
(n=28), \\
\text { Mean } \pm \text { SD } \%\end{array}$ & $\begin{array}{c}\text { QLF Group } \\
(n=23), \\
\text { Mean } \pm \text { SD } \%\end{array}$ & $P$ \\
\hline Upper right & $8.25 \pm 0.99$ & $12.1 \pm 1.09$ & .01 \\
Middle right & $17.6 \pm 0.59$ & $17.8 \pm 0.92$ & .84 \\
Lower right & $20.0 \pm 1.77$ & $19.7 \pm 1.69$ & .91 \\
Total right & $45.8 \pm 1.98$ & $49.6 \pm 1.88$ & .18 \\
Upper left & $12.4 \pm 0.62$ & $14.0 \pm 1.03$ & .17 \\
Middle left & $19.9 \pm 0.98$ & $19.9 \pm 1.19$ & .68 \\
Lower left & $21.4 \pm 1.08$ & $17.0 \pm 1.18$ & .01 \\
Total left & $54.2 \pm 1.98$ & $50.7 \pm 1.89$ & .21 \\
& & & \\
QLD $=$ quality lung data \\
IPF = idiopathic pulmonary fibrosis & & \\
\hline
\end{tabular}

Table 3. Diagnostic Power of Major Vibration Response Imaging Indices

\begin{tabular}{lccccc}
\hline \hline Parameter & Sensitivity & Specificity & $\begin{array}{c}\text { Youden's } \\
\text { Index }\end{array}$ & $\begin{array}{c}\text { Positive } \\
\text { Likelihood } \\
\text { Ratio }\end{array}$ & $\begin{array}{c}\text { Negative } \\
\text { Likelihood } \\
\text { Ratio }\end{array}$ \\
\hline Total score & 0.696 & 0.643 & 0.339 & 0.783 & 0.536 \\
MEF & 1.000 & 0.824 & 0.824 & 0.739 & 1.000 \\
$\begin{array}{l}\text { Abundant } \\
\text { crackles }\end{array}$ & 0.696 & 0.964 & 0.660 & 0.870 & 0.821 \\
MEF = maximal energy frame & & & & \\
\hline
\end{tabular}

likely that the highly restricted vibration energy shown in MEF images could have stemmed from restrictive ventilatory dysfunction. The upper lung lobes may theoretically have a priority for ventilation, which appeared inconsistent with our finding that less vibration energy was focused.

Another major finding of this study was that the VRI total score correlated positively with single-breath $\mathrm{D}_{\mathrm{LCO}}$ percent predicted, a parameter reflecting the diffusing ca- pacity, suggesting that subjects with poorer diffusing capacity are more likely to have aberrant VRI grading. Unfortunately, the fact that the total grade of VRI correlated with neither FVC percent predicted nor $\mathrm{FEV}_{1}$ percent predicted has rendered it difficult to interpret the results, inasmuch as these 2 indices clinically remain the major approaches for determining, at least physiologically, the severity of IPF.

The significance of VRI total score is compelling. Previous reports documented that subjects with pneumonia ${ }^{8}$ and $\mathrm{COPD}^{4}$ had a markedly higher total score than healthy individuals (see the supplementary materials at http:// www.rcjournal.com), suggesting that an increased total score might be an insensitive marker of pulmonary diseases. However, higher scores indicating more significant anomaly were negatively correlated with single-breath $\mathrm{D}_{\mathrm{LCO}}$ percent predicted. Further studies in a larger population are needed to determination whether the VRI total score may help predict the severity of IPF.

Although IPF subjects had slightly lower maximum vibration energy than healthy subjects, the difference between the 2 groups appeared statistically insignificant, possibly because of an insufficient number of subjects enrolled in the study. It is also likely that the maximum vibration energy has a limited diagnostic power for IPF.

QLD did not seem to be an appropriate diagnostic tool for IPF. This was in line with the fact that, in subjects with IPF, QLD failed to increase in bilateral lower lobes. The value of QLD remains to be further investigated in successive studies.

Of 23 subjects with IPF who had typical changes in MEF images in the present study, 6 did not exhibit evidence of restrictive ventilatory dysfunction. This led us to speculate that aberrant VRI indices might precede lung function decline, rendering VRI a more sensitive technique for IPF. MEF might be a candidate for the diagnosis of IPF, inasmuch as changes in MEF images were typically seen in subjects with IPF but not in healthy subjects. 
MEF images were deemed to be a critical index for diagnosis of IPF, particularly when combined with miscellaneous parameters, including dynamic image, EVP, or adventitious lung sounds. Furthermore, features of MEF images in IPF could be readily distinguished from those in COPD, asthma, and other common respiratory diseases. (See the supplementary materials at http://www.rcjournal. com.) This might be because of the capacity of VRI to display early-stage pulmonary physiological changes, which were considered to be the consequence of poor living environments and air pollution. Importantly, none of the healthy subjects showed changes in the MEF image typically witnessed in subjects with IPF. It was unlikely that the high sensitivity of the MEF curve, but not the remaining VRI indices, was related to the sample size in our study.

Several major limitations must be considered. First, our small sample size stemmed from the fact that IPF is a respiratory disease with a relatively low incidence compared with asthma and COPD. An increased sample size could render the distinct characteristics of IPF to be clearly displayed in different levels of disease severity. However, our major findings remain robust, as the typical VRI manifestations of IPF could be characterized, and the parameter with high diagnostic performance was captured. Second, comparison of VRI features between subjects with IPF and those with other respiratory diseases such as COPD might advance our understanding of which VRI indices more specifically reflect restrictive ventilatory dysfunction and poor diffusing capacity. Third, it would be helpful if comparisons were made between subjects with interstitial pulmonary fibrosis and those with miscellaneous subtypes of IPF. Finally, the increased VRI total score, as mentioned previously, might not specifically indicate the presence of IPF; an investigation into more miscellaneous specific indices is necessary.

In summary, the VRI technique may be helpful to discriminate between IPF subjects and healthy individuals. MEF and an abundance of crackles might serve as a diagnostic tool for IPF. Further studies that investigate the features of VRI in IPF subjects with different disease severities and treatment responses are indicated.

\section{ACKNOWLEDGMENTS}

We thank Ms Bing-jie Kang and Deep Breeze Ltd for providing the VRI lung system and technical training.

\section{REFERENCES}

1. Grenier P, Brauner M. Imaging of fibrosing interstitial pneumonias. Bull Acad Natl Med 2010;194(2):353-364; discussion 364-365.

2. Dunn TL, Watters LC, Hendrix C, Cherniack RM, Schwarz MI, King TE Jr. Gas exchange at a given degree of volume restriction is different in sarcoidosis and idiopathic pulmonary fibrosis. Am J Med 1988;85(2):221-224.

3. Wang Z, Jean S, Bartter T. Lung sound analysis in the diagnosis of obstructive airway disease. Respiration 2009;77(2):134-138.

4. Guntupalli KK, Reddy RM, Loutfi RH, Alapat PM, Bandi VD, Hanania NA. Evaluation of obstructive lung disease with vibration response imaging. J Asthma 2008;45(10):923-930.

5. Waseem M, Reynolds T, Jara F, Gat Merav, Rozen D, Pollack C. Vibration response imaging breathing profile in acute asthmatic subjects before and after treatment (abstract). Chest 2011;140:219A.

6. Dellinger RP, Parrillo JE, Kushnir A, Rossi M, Kushnir I. Dynamic visualization of lung sounds with a vibration response device: a case series. Respiration 2008;75(1):60-72.

7. Mor R, Kushnir I, Meyer JJ, Ekstein J, Ben-Dov I. Breath sound distribution images of subjects with pneumonia and pleural effusion. Respir Care 2007;52(12):1753-1760.

8. Bartziokas K, Daenas C, Preau S, Zygoulis P, Triantaris A, Kerenidi $\mathrm{T}$, et al. Vibration response imaging: evaluation of rater agreement in healthy subjects and subjects with pneumonia. BMC Med Imaging 2010;10(1):6.

9. Becker HD, Slawik M, Miyazawa T, Gat M. Vibration response imaging as a new tool for interventional-bronchoscopy outcome assessment: a prospective pilot study. Respiration 2009;77(2):179-194.

10. Cinel I, Jean S, Tay C, Gratz I, Deal E, Parrillo JE, Dellinger RP. Case report: vibration response imaging findings following inadvertent esophageal intubation. Can J Anaesth 2008;55(3):172-176.

11. Comce F, Bingol Z, Kiyan E, Tanju S, Toker A, Cagatay P, Ece T. Vibration-response imaging versus quantitative perfusion scintigraphy in the selection of subjects for lung-resection surgery. Respir Care 2011;56(12):1936-1941.

12. Demedts M, Costabel U. International multidisciplinary consensus classification of the idiopathic interstitial pneumonias. Am J Respir Crit Care Med 2002;19(5):794-796.

13. Miller MR, Hankinson J, Brusasco V, Burgos F, Casaburi R, Coates A, et al. Standardisation of spirometry. Eur Respir J 2005;26(2):319338.

14. MacIntyre NR, Crapo RO, Viegi G, Johnson DC, van der Grinten $\mathrm{CP}$, Brusasco V, et al. Standardisation of the single-breath determination of carbon monoxide uptake in the lung. Eur Respir J 2005; 26(4):720-735. 